\title{
Long term outcome of infective endocarditis in patients who were not drug addicts: a 10 year study
}

\author{
J C Castillo, M P Anguita, A Ramírez, J R Siles, F Torres, D Mesa, M Franco, I Muñoz, \\ $M$ Concha, F Vallés
}

\begin{abstract}
Objective-To determine the clinical features and long term prognosis of infective endocarditis in patients who were not drug addicts.

Design-Prospective case series.

Setting-A university hospital that is both a referral and a primary care centre.

Patients-138 consecutive cases of infective endocarditis diagnosed and treated from January 1987 to March 1997.

Results-Mean patient age was 44 (20) years old. 95 patients (69\%) had native valve endocarditis and $43(31 \%)$ had prosthetic valve endocarditis. Staphylococci were the causal microorganisms in 34\% of cases and streptococci in 33\%. Severe complications occurred in $83 \%$ of patients and $51 \%$ of patients underwent surgery during the active phase ( $22 \%$ was emergency surgery). Inpatient mortality was $21 \%$. During a follow up of $56(44)$ months, 10 patients $(9 \%)$ needed late cardiac surgery and seven ( $5 \%$ of the whole series) died. Overall 10 year survival was $71 \%$. There were no significant differences in survival depending on the type of treatment received during the hospital stay (medical or combined medical-surgical).

Conclusions-A high early surgery rate is related to good long term results and does not increase in-hospital mortality. Medical treatment, however, also offers favourable long term results in cases of responsive infective endocarditis where poor prognostic factors are absent. (Heart 2000;83:525-530)
\end{abstract}

Keywords: infective endocarditis; staphylococci; streptococci

Untreated infective endocarditis is one of the few universally fatal infectious diseases. The poor prognosis of the disease is not only attributable to the considerable morbidity and mortality during the active phase of the disease, but also to late complications and sequelae occurring once the infection has been eradicated..$^{12}$ Prognosis in patients surviving the initial stages of infective endocarditis is dependent on three main factors: substantial risk of recurrence (recently estimated at $0.3-2.5 / 100$ patient years); the need for valve replacement during follow up as a result of valve lesions caused or exacerbated by endocarditis; and, lastly, death particularly from heart failure. ${ }^{34}$

As a result of advances in diagnosis, improved antimicrobial treatment, and earlier detection and management of complications, the short term prognosis for this disease has improved over the past few years. Improvements in microbiology techniques, together with the recent introduction and development of transoesophageal echocardiography, have made a substantial contribution to ensuring early diagnosis, providing accurate information on possible complications and their monitoring. ${ }^{5-7}$ Very few series, however, refer to long term survival in infective endocarditis in the era of transoesophageal echocardiography. ${ }^{6} 8$

We conducted a prospective study involving cases of infective endocarditis in nonintravenous drug users and their short and long term survival.
Patients and methods

A prospective study was conducted involving all consecutive cases of infective endocarditis in non-intravenous drug users diagnosed and treated at our centre between January 1987 and March 1997. Our hospital is a tertiary centre providing health care cover for both the local population (480 000 inhabitants) and the surrounding area, making a total referral population of 1389000 inhabitants.

The following data were recorded for each patient: age, sex, predisposing heart disease, evaluation of the predisposing heart disease, site of infection, type of endocarditis, valve involved, causal organism, echocardiographic findings, cardiac and extracardiac complications, treatment given during hospitalisation, surgical requirements, and mortality. Recurrence of the disease, the need for surgery, and death were evaluated during the follow up period.

Until 1994, all cases of infective endocarditis were diagnosed on the basis of the criteria proposed by Von Reyn and colleagues ${ }^{9}$ and on the criteria of Durack and colleagues ${ }^{10}$ thereafter. All intravenous drug users were excluded from the study. Early prosthetic valve endocarditis (PVE) was considered when the episode occurred in the first two months following heart surgery, while intermediate and late PVE was diagnosed when the episode developed 2-12 months and more than one year after surgery, respectively. Transoesophageal echocardiography has been available in our hospital 
since 1990. All patients received antibiotic treatment in accordance with the susceptibility of the causal organism. Treatment was administered for 4-6 weeks according to both the causal agent and the type of infective endocarditis involved. Surgical treatment was indicated during hospitalisation in the following circumstances: moderate to severe heart failure; significant valve regurgitation with major ventricular volume overloading; repeated major emboli (two or more); severe mechanical complications; in episodes caused by virulent organisms which cannot be eradicated by antibiotic treatment alone (fungi, Brucella species, Coxiella species); in cases of early PVE; endocarditis on pacemaker leads; and in cases of persistent sepsis despite theoretically adequate antibiotic treatment. Emergency surgery was defined as surgery for patients with life threatening conditions that could not be postponed for more than 24 hours, and elective surgery comprised those interventions that could be delayed for longer time periods without risk to the patient.

All survivors to the in-hospital phase were included in the prospective follow up. Blood cultures, one and two months postdischarge, were performed with a clinical check up and an echocardiographic study to assess anatomy and any haemodynamic sequelae of the disease. When blood cultures were negative two months after discharge, the infection was considered to be cured. Death occurring during the six weeks postdiagnosis was considered as early mortality. A positive blood culture for the same causal organism two months after discharge was classed as relapse, while recurrence was taken to be a new episode of infective endocarditis caused by a different organism or by the same organism as the initial infection but arising over two months postdischarge. During follow up, some patients required valve replacement either because of the course of their underlying heart disease or because of the haemodynamic repercussions of valve lesions caused by the infection.

STATISTICAL ANALYSIS

All qualitative variables are expressed as percentage and quantitative variables as mean (SD). The differences between patient groups have been evaluated by Fisher's exact test or $\chi^{2}$ test for qualitative variables, while Student's $t$ test was used for unpaired data in the case of quantitative variables.

Overall survival curves (including early mortality) and long term survival curves for patients surviving the active phase are given. The Kaplan-Meier actuarial method was used for the survival curves. Comparison of the survival curves of the different subgroups was made using the Mantel log rank test. Values of $\mathrm{p}<0.05$ were considered significant.

\section{Results}

GENERAL CHARACTERISTICS

Our series comprises 138 cases of infective endocarditis in 126 non-intravenous drug users. The diagnosis of infective endocarditis was made according to the aforementioned
Table 1 General characteristics in 138 cases of infective endocarditis in non-drug addict patients

\begin{tabular}{ll}
\hline & $\begin{array}{l}\text { Number of patients } \\
(\%)\end{array}$ \\
\hline Age (years) & $41(21)^{\star}$ \\
NVE & $51(16)^{\star}$ \\
PVE & $1.7 / 1$ \\
Sex (male/female) & $2.3 / 1$ \\
NVE & $95(69)$ \\
PVE & $43(31)$ \\
NVE & $8(19)$ \\
PVE & $14(32)$ \\
Early & $21(49)$ \\
Intermediate & $29(21)$ \\
Late & $52(38)$ \\
Underlying heart disease & $29(21)$ \\
No & $28(20)$ \\
Rheumatic & \\
Congenital & $59(43)$ \\
Degenerative & $59(43)$ \\
Location of infection & $20(14)$ \\
Mitral & \\
Aortic & Pther
\end{tabular}

${ }^{\star} \mathrm{SD}$; NVE, native valve endocarditis; PVE, prosthetic valve endocarditis.

criteria. The diagnosis of infective endocarditis was definite in 124 cases (pathologically definite in 70 cases - those who underwent surgery-and clinically definite in 54 cases). Fourteen patients $(10 \%)$ were classified as possible endocarditis. When retrospectively applying the Duke criteria, none of our patients was rejected. Mean patient age was 44 (20) years, with ages ranging from 2 months to 72 years. Thirteen of the cases were patients under the age of 14 years. Ninety five $(69 \%)$ cases were native valve endocarditis (NVE) and 43 (31\%) cases were PVE (table 1).

A presumed portal of entry was identified for $38 \%$ of the cases. Dental procedures had been performed in 13 patients (9\%). In four cases (3\%) the portal of entry was the genitourinary tract (three cases of prostatectomy and one case of urinary catheter related infection) and in three cases $(2 \%)$ it was the digestive tract (two patients with colon cancer and one with active ulcerative colitis). Other entry routes were: cutaneous abscesses caused by Staphylococcus aureus in two patients; intravenous catheters in eight patients; heart surgery, which was performed 7-10 days before the start of infective endocarditis symptoms in six patients (prosthetic valve replacement in five and correction of tetralogy of Fallot in one patient); and haemodialysis in six patients. Predisposing heart disease was present in 68 patients with NVE (80\%) (table 1). The most common heart condition in NVE was aortic regurgitation $(10 \%)$, followed by mitral valve prolapse, aortic stenosis, and congenital heart disease ( $8 \%$ each). Infection occurred on the left side of the heart in $86 \%$ of the cases, with no significant differences between the mitral and aortic valves. A previous infective endocarditis event was more common in PVE than in NVE patients $(23 \% v 2 \%, \mathrm{p}<0.001)$. Fifty eight patients $(42 \%)$ were referred to our institution because of infective endocarditis or suspicion of infective endocarditis. The median duration of the first symptom before diagnosis was shorter in local patients (26 days, range 7-130 
Table 2 Infective microorganisms in 138 cases of endocarditis in non-drug addict patients

\begin{tabular}{llll}
\hline & \multicolumn{2}{l}{ Number of cases (\%) } \\
\cline { 2 - 4 } & NVE & 1 year PVE & Late PVE \\
\hline Staphylococci & $37(39)$ & $6(27)$ & $4(19)$ \\
S aureus, methicillin susceptible & $32(34)$ & $2(9)$ & $2(10)$ \\
Coagulase negative staphylococci & $5(5)$ & $4(18)$ & $2(10)$ \\
"Viridans" streptococi & $20(21)$ & $2(9)$ & $8(38)$ \\
Enterococci, vancomycin susceptible & $10(11)$ & $3(14)$ & - \\
Corynebacterium species & $6(6)$ & $3(14)$ & $1(5)$ \\
Brucella & $3(3)$ & - & $5(24)$ \\
Coxiella burnetii & $2(2)$ & - & $1(5)$ \\
Fungi & $2(2)$ & $2(9)$ & $1(5)$ \\
Other† & $3(3)$ & $2(9)$ & $1(5)$ \\
Culture negative & $11(12)$ & $4(18)$ & \\
\hline
\end{tabular}

${ }^{\star}$ Candida albicans (3), Torulopsis glabrata (1), Saccharomyces (1)

$\dagger$ Listeria monocytogenes, Haemophilus, Acinetobacter, and Escherichia coli

days) than in referred patients (37 days, range $20-210$ days) $(\mathrm{p}<0.05)$.

Table 2 shows the aetiology of infective endocarditis. No causal organism was found in 16 patients ( 11 patients in the first half of the study and five in the last five years, $\mathrm{p}<0.1$ ). Twelve of these patients $(75 \%)$ had received antibiotics in the week before blood culturing. Staphylococcus was the most frequent organism responsible in the NVE group, while in PVE cases streptococcal infection was more common.

There were four cases of infective endocarditis on pacemaker leads. In one case an abscess at the tip of the lead in the right ventricle implant site was detected. In the rest, echocardiography showed bacterial growth on the lead around the tricuspid valve. All episodes were caused by $S$ aureus. Three patients were operated on in the active phase. Two of them had a favourable outcome and the third patient died from postoperative malignant ventricular arrhythmias. The fourth patient refused surgery but had a favourable outcome with medical treatment only.

\section{ECHOCARDIOGRAPHIC DATA}

All patients were examined by transthoracic echocardiography and, from 1990 onwards, the last 94 patients were also examined by transoesophageal echocardiogram (68\%). Echocardiography detected vegetations in $86 \%$ of the cases $(59 \%$ with transthoracic echocardiography and $92 \%$ with transoesophageal echocardiography, $\mathrm{p}<0.01)$ This difference was greater in the PVE group $(24 \%$ v $81 \%$, $\mathrm{p}<0.001)$ than in NVE group $(75 \% v 97 \%$, $\mathrm{p}<0.05)$. When only those cases that were verified at surgery were taken into consideration, transthoracic echocardiography gave a sensitivity of $66 \%$ compared with $100 \%$ sensitivity for transoesophageal echocardiography $(\mathrm{p}<0.01)$. Sensitivity was similar for mitral and aortic valve infective endocarditis. Beside the vegetations, echocardiography revealed further findings or complications in 30 patients $(22 \%)$. Surgery was indicated during the active phase according to the echocardiographic findings in all these patients (table 3 ).

IN-HOSPITAL COMPLICATIONS

Cardiac and extracardiac complications were a frequent finding $(83 \%)$. The most common complication was the onset of heart failure
Table 3 Other echocardiographic findings in a series of 138 cases of infective endocarditis in non-drug addict patients

\begin{tabular}{lc}
\hline & $\begin{array}{l}\text { Number of } \\
\text { patients }\end{array}$ \\
\hline Periaortic abscess & 6 \\
Mitral abscess & 2 \\
Interventricular septum abscess & 1 \\
Rupture of ASV with shunt to right chambers & 2 \\
Rupture of interventricular septum with VSD & 1 \\
Prosthetic obstruction & 1 \\
Severe prosthetic dehiscence & 6 \\
Severe valvar regurgitation & 17 \\
Mural thrombus probably infected & 3 \\
\hline
\end{tabular}

ASV, aneurism of the sinus of Valsalva; VSD, ventricular septal defect.

Table 4 Cardiac and extracardiac complications in both types of infective endocarditis in a series of 138 cases in non-drug addict patients

\begin{tabular}{llll}
\hline & $\begin{array}{l}\text { NVE } \\
n=95(\%)\end{array}$ & $\begin{array}{l}\text { PVE } \\
n=95(\%)\end{array}$ & $p$ Value \\
\hline Heart failure & $38(40)$ & $18(42)$ & NS \\
Embolisms & $30(32)$ & $12(28)$ & NS \\
CNS complications & $20(21)$ & $8(19)$ & NS \\
Persistent sepsis & $18(19)$ & $8(19)$ & NS \\
Renal failure & $12(13)$ & $5(12)$ & NS \\
Metastasic abscesses & $13(14)$ & $3(7)$ & NS \\
Mycotic aneuryms & $4(4)$ & $4(9)$ & NS \\
Inmunologic disorders & $74(78)$ & $28(65)$ & NS \\
\hline
\end{tabular}

*Includes glomerulonephritis (10 cases), cutaneous vasculitis (7 cases) and altered inmunologic parameters (raised rheumatoid factor and circulating immune complexes levels, and low C3 and $\mathrm{C} 4$ concentrations)

NS, not significant; CNS, central nervous system.

(40\%), followed by embolism (30\%), neurological disorders (20\%), persistent sepsis $(19 \%)$, acute renal failure $(12 \%)$, metastasic abscesses (11\%), and mycotic aneurysms (6\%). There were no differences between NVE and PVE as regards complications (table 4).

\section{TREATMENT}

All patients received antibiotic treatment. Forty seven patients with NVE (50\%) and 23 patients with PVE (53\%) underwent surgery during the active phase. Elective surgery predominated over emergency procedures $(29 \%$ v $22 \%)$. Seven patients $(5 \%)$ died on the day of admission before receiving any treatment. The most common indication for surgery was congestive heart failure $(48 \%)$, followed by persistent sepsis (26\%). Other indications included underlying heart disease (7\%), repeated embolism (4\%), glomerulonephritis with acute renal failure despite antibiotic treatment $(4 \%)$, and the causal organism $(2 \%)$. Surgery was more frequently required in aortic infective endocarditis (40/59 patients, $67 \%)$ than in mitral infective endocarditis $(22 / 59$ patients, $38 \%)(\mathrm{p}<0.05)$.

The most frequent cause contraindicating surgery during the hospitalisation stage was a good response to medical treatment alone or a favourable prognosis a priori $(27 \%)$, patient age $(8 \%)$ or any formal contraindication to surgery $(3.5 \%)$. There was one case of patient refusal and one involving a patient diagnosed with a carcinoma of the colon with endocarditis involving a pacemaker lead, who refused any kind of surgery but who later responded well to antimicrobial treatment. 


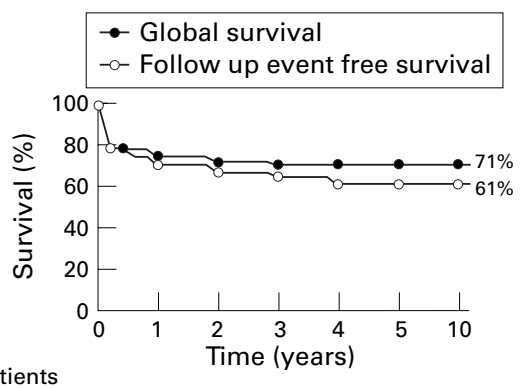

No. of patients

at risk

$\begin{array}{llllllll}\text { Global survival } & 138 & 83 & 71 & 60 & 49 & 38 & 12\end{array}$

$\begin{array}{lllllll}\text { Follow up event } 138 & 81 & 67 & 54 & 42 & 31 & 5\end{array}$ free survival

Figure 1 Kaplan-Meier survival curves. Follow up event free survival in a series of 138 cases of infective endocarditis.

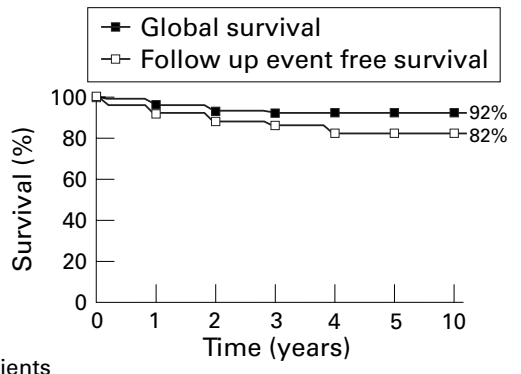

No. of patients

at risk

$\begin{array}{llllllll}\text { Global survival } & 109 & 83 & 71 & 60 & 49 & 38 & 12\end{array}$

$\begin{array}{llllllll}\text { Follow up event } & 109 & 81 & 67 & 54 & 42 & 31 & 5\end{array}$ free survival

Figure 2 Kaplan-Meier survival curves. Follow up event free survival in 109 survivors to the active phase.

EARLY MORTALITY

Twenty nine patients $(21 \%)$ died in hospital (19 patients $(20 \%)$ with NVE and 10 patients (23\%) with PVE, no significant difference). The highest mortality rate was registered among early PVE with four of the eight cases dying $(50 \%)$. Four of the 14 intermediate PVE cases $(28 \%)$ died as did two of the 20 late PVE patients (10\%). Mortality was $20 \%$ among patients receiving antibiotic treatment alone and $21 \%$ for patients in the combined medical-surgical treatment group. The seven patients who died during the first 24 hours after admission and who received no treatment at all have been included in the antibiotic treatment group. The most common cause of early mortality was of surgical origin $(45 \%)$, followed by heart failure and cardiogenic shock $(24 \%)$, brain haemorrhage $(14 \%)$, postoperative pneumonia $(7 \%)$, sepsis $(6 \%)$, and pulmonary embolism $(3.5 \%)$.

Mortality was $23 \%$ in the staphylococcal endocarditis group, $14 \%$ in the streptococcal group, and $16 \%$ in the other microorganism group. Eight of the 15 patients in which the causal agent could not be identified also died. The causal agent was identified in only $69 \%$ of the patients who died during the hospitalisation stage, compared with $94 \%$ of the survivors $(\mathrm{p}<0.001)$. Other short term bad prognostic factors were older age $(p<0.01)$, the onset of cardiac complications $(\mathrm{p}<0.01)$, and the need for emergency surgery $(p<0.05)$. Prognosis

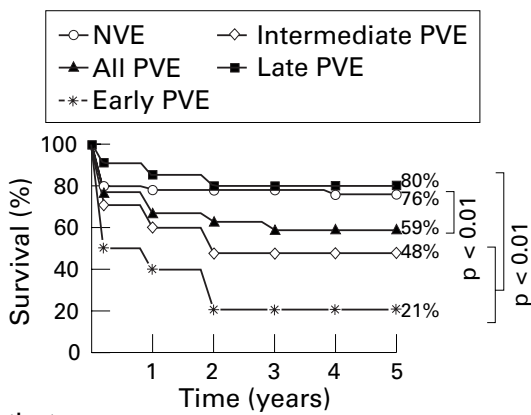

No. of patients

at risk

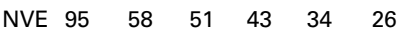

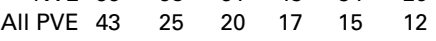

$\begin{array}{lllllll}\text { Early PVE } & 8 & 3 & 1 & 1 & 1 & 1\end{array}$

Intermediate $\begin{array}{llllll}14 & 6 & 4 & 3 & 2 & 2\end{array}$

PVE

$\begin{array}{lllllll}\text { Late PVE } & 21 & 16 & 15 & 13 & 12 & 9\end{array}$

Figure 3 Overall survival curves according to the type of infective endocarditis. Differences between native (NVE) and prosthetic (PVE) valve endocarditis, and between late and one year PVE, are significant.

was not affected by the type of infective endocarditis (NVE or PVE) or by site and causal agent.

FOLLOW UP

The average follow up for the 109 patients surviving the active phase was 56 (44) months (range 12-135 months).

Overall survival (including mortality during the in-hospital phase) was $75 \%$ at one year and $71 \%$ at five and 10 years (fig 1). Excluding those patients who died during the active phase, one year survival was $96 \%$ with a five and 10 year survival rate of $92 \%$. Follow up event free survival at one year was $92 \%$, and $82 \%$ at five and 10 years (fig 2). Overall survival for NVE was $78 \%$ at one year and $76 \%$ at five years, and for PVE it was $67 \%$ and $59 \%$, respectively $(p<0.01)$. In the cases of late PVE, one year survival was $85 \%$ and five year survival was $80 \%$ (fig 3). One year and five year survival rates for NVE in survivors to the active phase were $98 \%$ and $96 \%$, while in PVE they were $90 \%$ and $82 \%$, respectively ( $p<0.05$ ) (fig $4)$. Regarding the type of treatment received during hospitalisation, follow up event free survival was similar in both groups (medical and surgical) (fig 5).

There was recurrence of infective endocarditis in seven of the 109 survivors (6\%). Four of these seven patients also underwent surgery (two of these infective endocarditis cases were caused by $Q$ fever). Of the remaining three patients, one died before surgery because of a brain haemorrhage while the other two responded satisfactorily to medical treatment alone. There was no relapse.

Ten patients $(9 \%)$ required late surgery during follow up, and only one patient $(1.4 \%)$ required a second operation (because of a severe regurgitation leak). In most cases, surgery was performed in the first two years following the infective endocarditis. All presented a favourable postoperative course, and no deaths occurred. Eight of these 10 patients received valve replacements while the remaining two underwent a percutaneous procedure 


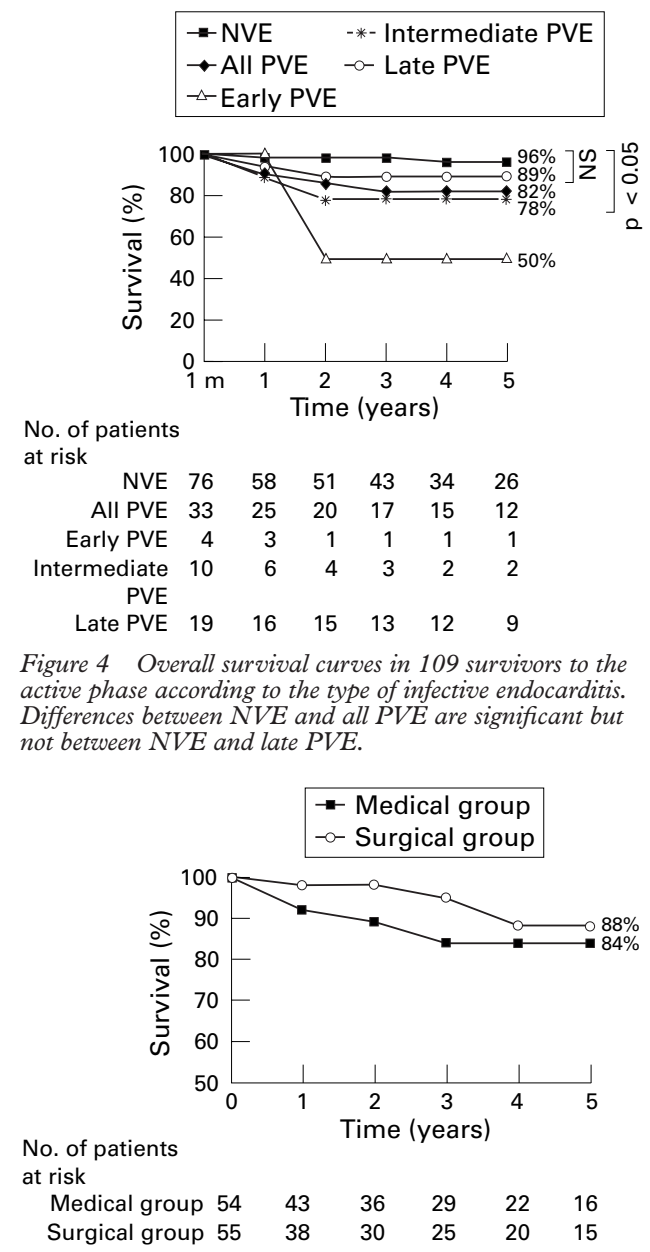

Figure 5 Follow up event free survival curves in 109 survivors to the active phase depending on the treatment (medical or combined medical-surgical) received in the active phase. No significant differences in survival were observed.

(percutaneous mitral valvuloplasty in two patients with rheumatic mitral stenosis). Surgery was performed on six of the patients because of progression of the underlying heart disease, while the indication for surgery in the remaining four patients was the recurrence of the infective endocarditis.

Seven patients died during follow up (5\% of the total in the series), mainly during the first two years after the episode. In two of these cases, death was related to a new episode of infective endocarditis; in a further two cases (one of which also involved prior severe left ventricular dysfunction) the cause was sudden death. One patient, who had refused surgery, died from a brain haemorrhage caused by a ruptured mycotic aneurysm, while a further two patients died from congestive heart failure associated with severe pulmonary hypertension.

At the end of the follow up period, 89 patients were alive and free of events- $64 \%$ of the series.

\section{Discussion}

This 10 year study, comprising 138 cases of infective endocarditis in non-drug addict patients treated at our hospital, is similar in size to other series recently reported..$^{11-14}$ Possible referral bias affecting series like ours, conducted in tertiary hospitals, should not be overlooked.

Complications of the infective endocarditis during the active phase were very frequent, occurring in $83 \%$ of the cases. These figures, similar to those reported by other authors, ${ }^{121516}$ reflect the diversity of clinical manifestations and the severity of the disease. As a general rule, complications in NVE are less frequent. In our series, however, rates were similar to PVE complications, probably related to aggressive microorganisms, a nosocomial origin, or to the delay in diagnosing the disease.

Our series shows a high incidence of surgery performed during the active phase of the disease $(51 \%)$, regardless of the type of infective endocarditis, and on an elective basis, following stabilisation of the condition. Seven patients died in the first few hours after admission before surgery could be considered. The delay in diagnosing the disease is an important factor affecting the high rate of surgery. A delayed diagnosis may either be caused by non-specific initial symptoms, by ignorance of some of the relatively common causal agents in our area (for example, Coxiella burnetti) or by the unavailability of transoesophageal echocardiography at all hospitals in the area. A delay in diagnosis and therefore in the institution of antimicrobial treatment is associated with a higher rate of complications, thus requiring a greater number of surgical procedures. Comparing our incidence of surgery performed during the active phase with those reported in other series, ${ }^{6}{ }^{17-19}$ early elective surgery is performed more frequently at our centre - that is, once the disease has been stabilised by instituting appropriate antibiotic treatment. Trends in literature over the past two decades, however, reveal an increase in the number of patients undergoing surgery during the active phase. For example, in the series of Von Reyn and colleagues, ${ }^{9} 18 \%$ of patients underwent surgery. In the series of Malquarti and colleagues, ${ }^{20}$ conducted between 1970 and $1982,26 \%$ of NVE cases were operated. More recent publications report rates for surgery of $15-34 \% .^{68}{ }^{18}$ In the series presented here, 50\% of NVE and $53 \%$ of PVE cases underwent surgery during the active phase.

Unlike in other studies, ${ }^{6}{ }^{13} 14$ the most frequent cause of early mortality is not heart failure or persistent sepsis but is surgical in origin, related to a high number of early surgical procedures. Most deaths occur in cases of emergency surgery - that is, in haemodynamically unstable patients with severe complications. Patients who only received medical treatment, however, responded favourably and did not, therefore, require surgery. In the light of these factors, the results for each of the two groups are not easily comparable, since a favourable clinical course would give a bias towards the medical treatment group; however, those patients who died during the first few hours after admission and therefore did not undergo surgery have also been included to counteract bias. Higher mortality was seen in 
cases where the causal agent was not identified ( $50 \%$ of patients with negative blood cultures). Other factors indicating early mortality were age, the presence of cardiac complications, persistent sepsis, and the need for emergency surgery. There was no significant difference in mortality between patients following medical treatment $(20 \%)$ or those undergoing surgical treatment $(21 \%)$. Although they vary from one series to another, surgical mortality rates have tended to decrease over recent decades owing to technological developments, with rates as low as $14 \%$ being recently reported..$^{21}$

In the very few studies dealing with follow up on infective endocarditis, a large number of patients required late surgery, which contrasts with our findings. In the reports mentioned above, late surgery rate ranged from $27-47 \% .^{8}{ }^{18} 19$ In our series, with a mean follow up of 56 months, only 10 patients ( $9 \%$ of the survivors) underwent late surgery as patients had undergone elective surgery during the active phase of the disease. Only one patient had to be reoperated on. Low late surgery rate may be related to low late mortality rate $(5 \%)$ which is lower than those reported by other authors. At the end of the follow up period, $67 \%$ of patients were alive and event free, which contrasts with figures of $22-38 \%$ reported by others. ${ }^{8} 18$

Overall survival (including in-hospital mortality) in the series of Delahaye and colleagues, ${ }^{3}$ conducted between 1970 and 1982, was 57\% at five years while our series yields an overall five year survival rate of $71 \%$. When related to the type of infective endocarditis involved, in the series of Calderwood and colleagues, ${ }^{19}$ conducted between 1975 and 1983, the five year survival rate for PVE was $60 \%$, while the rate in our series is $82 \%$. In the series of Tornos and colleagues ${ }^{18}$ the five year survival rate for NVE was $88 \%$ and for late PVE $80 \%,{ }^{8}$ while in our series the rates were $96 \%$ and $82 \%$, respectively. More recently Schultz and colleagues, ${ }^{6}$ in their series conducted between 1989 and 1993, report a one year survival rate for survivors to the active phase of $73 \%$ in NVE and $83 \%$ in late PVE, compared with rates of $98 \%$ and $94 \%$ in our series. When comparing the type of treatment received during the active phase (medical or combined medicalsurgical), the overall, event free survival rate in the surgical group was slightly higher than in the medical group (no significant differences).
In conclusion, our results seem to indicate that a high elective surgery rate in the active phase of infective endocarditis improves long term survival rate without increasing early mortality. However, in cases of responsive infective endocarditis with absence of poor prognostic factors, medical treatment also offers favourable long term results.

1 Scheld WM, Sande MA. Endocarditis and intravascular infections. In : Mandell GL, Douglas RG, Benett JE, eds. Principles and practice of infectious diseases, 3rd ed. New York: Churchill Livingstone; 1990:670-706.

2 Korzeniowski OM, Kaye D. Infective endocarditis. In: Heart disease, a textbook of cardiovascular medicine. Braunwald E, ed. Philadelphia: WB Saunders, 1992:1078-105.

3 Delahaye F, Ecochard R, De Gevigney G, et al. The long term prognosis of infective endocarditis. Eur Heart $\mathcal{F} 1995$; 16(suppl 2):48-53.

4 Tornos MP, Almirante B, Soler J. Natural history and prognosis in infective endocarditis. Rev Esp Cardiol 1998; 51(suppl 2):40-3.

5 Cormier B, Vahanian A. Echocardiography and indications for surgery. Eur Heart $\mathcal{F}$ 1995;16(suppl B):68-71.

6 Schulz R, Werner GS, Fuchs JB, et al. Clinical outcome and echocardiographic findings of native and prosthetic valve endocarditis in the 1990s. Eur Heart $\mathcal{F} 1996 ; 17: 281-8$.

7 Werner GS, Schulz R, Fuchs JB, et al. Infective endocarditis in the elderly in the era of transoesophageal echocardiography: Clinical features and prognosis compared with younger patients. Am f Med 1996;100:90-7.

8 Tornos P, Almirante B, Olona M, et al. Clinical outcome and long-term prognosis of late prosthetic valve endocarditis: a 20-year experience. Clin Infect Dis 1997;24:381-6.

9 Von Reyn CF, Levy BS, Arbeit RD, et al. Infective endocarditis: an analysis based on strict case definitions. Ann Intern Med 1981;94:505-17.

10 Durack DT, Lukes AS, Bright DK, et al. New criteria for diagnosis of infective endocarditis: utilization of specific echocardiographic findings. Am 7 Med 1994;96:200-9.

11 Delahaye F, Goulet V, Lacassin F, et al. Characteristics of infective endocarditis in France in 1991. A year survey. Eur Heart f 1995;16:394-401.

12 Van der Meer J, Thompson J, Valkenbrug H, et al. Epidemiology of bacterial endocarditis in the Netherlands. I. Patients characteristics. Arch Intern Med 1992;152:1863-8.

13 Hogevik H, Olaison L, Rune A, et al. Epidemiologic aspects of infective endocarditis in an urban population: a 5-year prospective study. Medicine 1995;74:324-39.

14 Sandre R, Shafran SD. Infective endocarditis: review of 135 cases over 9 years. Clin Infect Dis 1996;22:276-86.

15 Hermans PE. The clinical manifestations of infective endocarditis. Mayo Clin Proc 1982;57:15-21.

16 Ivert TSA, Dismukes WE, Cobbs CG, et al. Prosthetic valve endocarditis. Circulation 1984;69:223-32.

7 Horstkotte D, Piper C, Niehues R, et al. Late prosthetic valve endocarditis. Eur Heart $\mathcal{7}$ 1995;16(suppl B):39-47.

18 Tornos MP, Permanyer-Miralda G, Olona M, et al. Long term complications of native valve infective endocarditis in non addicts. Ann Intern Med 1992;117:567-72.

19 Calderwood SP, Swinsky LA, Karchmer AW, et al. Prosthetic valve endocarditis. Analysis of factors affecting outcome of therapy. F Thorac Cardiovasc Surg 1986;92:77683.

20 Malquarti V, Saradarian W, Etienne J, et al. Prognosis of native valve infective endocarditis: a review of 253 cases. Eur Heart $\mathcal{F}$ 1984;5:11-20.

21 Mullany CJ, Chua YL, Schaff HV, et al. Early and late survival after surgical treatment of culture positive active endocarditis. Mayo Clin Proc 1995;70:517-25.

22 Acar J, Michel PL, Varenne O, et al. Surgical treatment of infective endocarditis. Eur Heart $\mathcal{F}$ 1995;16(suppl B):94-8. 